\title{
Drowsiness Detection Using Eye Blink and Facial Features Image Analysis
}

\author{
Ajay S. ${ }^{1}$, Azariah John K. ${ }^{1}$ R. Subhashini ${ }^{2}$, Joshua Thomas ${ }^{3}$ \\ ${ }^{\text {IStudent, }}{ }^{2}$ Professor, Deartment of Information Technology, Sathyabama Institute of Science and Technology, \\ Chennai, India, ${ }^{3}$ Senior Lecturer, Department of Computing, UOW Malaysia KDU Penang University College, \\ Malaysia
}

\begin{abstract}
Drowsiness detection and alert system is developed using OpenCV library and deep learning algorithms and implemented with a night vision camera and a computer to detect if a person is drowsy. The system uses a camera to capture the person's face and eyes to detect fatigue. In such a case where fatigue is detected, a warning signal is issued to alert the person and an SMS to a person related to the victim.
\end{abstract}

Keywords: EAR (Eye Aspect Ratio), Fatigue, Drowsiness, Blink Detection, SMS alert.

\section{Introduction}

Drowsiness is the state of feeling tired or sleepy. It mostly occurs when a person has lack of sleep or is tired. Drowsiness can lead to many other consequences if it is not taken care properly ${ }^{[7,8]}$. Health wise it might lead to forgetfulness or falling asleep at inappropriate times. Some researchers say Driving drowsy is considered more dangerous than driving on alcohol ${ }^{[2,3]}$. Drowsiness of a person not only affects the person drowsy; it might also affect the people around them. In a workplace when an employee is drowsy and sleeps, it affects the organization and the person. If the person feels drowsy and sleeps during work, it can lead to any accident, which might interrupt the orderly progress of the work. Drowsiness also may lead to breach of security in many places if the security personnel at work gets drowsy.

Our objective is to provide an alert system to this problem. In this manner the person and the person concerned to the victim can be alerted before it's late. To accomplish this, we will need a system, which will track the face and eyes of a person to check if drowsy, and sound an alarm to the victim and send an SMS to the person concerned to the victim.

The purpose of this study is to provide a real time monitoring using face and eye detection techniques. The video will capture with camera after all the analysis the victim who is drowsy and the person concerned with the victim will be warned separately.

Proposed Method: Existing drowsiness detection systems are only focused on driver drowsiness but the proposed system can be used for general drowsiness detection and can also send SMS unlike other drowsiness detection system ${ }^{[4,5]}$. Generally, drowsiness detection systems use different method to analyze drowsiness. Some method use the position of the head to analyze drowsiness, some use yawns to detect drowsiness, and some method use visual information of the eyes to analyze drowsiness ${ }^{[6]}$. Commonly most algorithms use face and eye movements to detect drowsiness. We propose a system which is cost efficient and has additional features for alerting the person and the person concerned with the victim. Our system finds the face and eye movements from the web camera to determine the state of the person's eyes and if drowsy. The alert system in this advanced than others since it not only warns the person, it also sends a SMS to the concerned person of the victim.

System Overview: Detection of drowsiness is done using computer vision and deep learning algorithms and Twilio api is used for SMS alert. The architecture is described as shown in Fig 1. The program flow of this projected system is: 


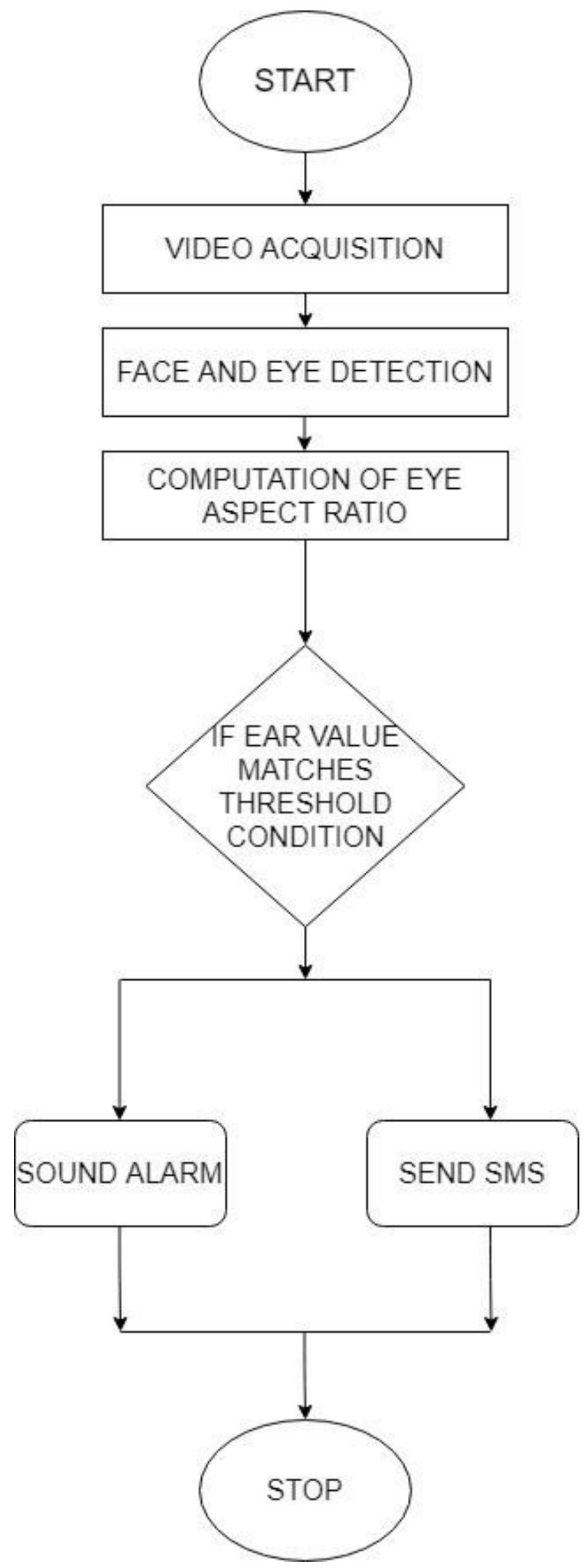

Fig. 1. Architecture of System Flow
A. Algorithms: The proposed system uses a combination of dlib's library, Eye aspect ratio algorithm $^{[1]}$ and Twilio SMS api.

Step 1: Getting input video stream from the webcam.

Step 2: Detecting face using dlib's pre trained hog face detector.

Step 3: Apply dlib's facial landmark predictor on the face and extract eye region.

Step 4: Calculating Ear value using the 6 points.

Step 5: If Ear threshold value is satisfied go to step 7.

Step 6: Go to step 2.

Step 7: Alert the person with warning sound and send SMS to the concerned person.

B. Working of the System: The system starts with the initialization process and acquires the face and eyes from the video stream. Then the system performs detection to extract the face and eye regions. For each frame we identify face and get the facial points and the coordinates of the points of eyes from dlib's facial landmark predictor. After localizing the eyes and finding the points, we compute a value called eye aspect ratio (EAR) ${ }^{[1]}$ to find if the person blinks or not, and check with the threshold if the person has closed the eyes for sufficiently long time to know if drowsy or not? If the value goes below the threshold, we sound an alarm and send a SMS to the concerned person.

C. Eye Aspect Ratio: It is a mathematical formula which gives an elegant solution to find the blinks of a person. After finding the landmarks of the eyes, the $6(\mathrm{x}, \mathrm{y})$ points are used to compute the aspect ratio between the height and width of the eye. The value of the 'EAR' ${ }^{[1]}$ value becomes less than 0.1 when the eyes are closed. Equation 1 is used to calculate the 'EAR' value using the 6 points (p1, p2, p3, p4, $\mathrm{p} 5, \mathrm{p} 6)$ which is depicted in figure 2.

$$
E A R=\frac{|| p 2-p 6||+|| p 3-p 5||}{2|| p 1-p 4||}
$$

This equation (1) finds the vertical and horizontal distance between the eye. The numerator calculates the vertical distance between the eye and denominator computes the horizontal distance between the eye. 


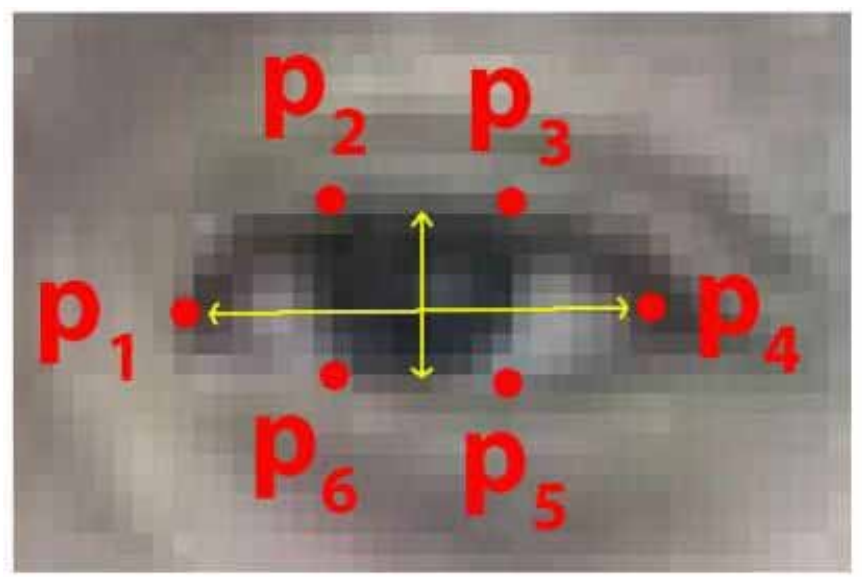

Fig. 2. Points of eye landmarks

Fig. 2 shows the 6 eye landmark points of the 68 dlib's facial landmark points detector.
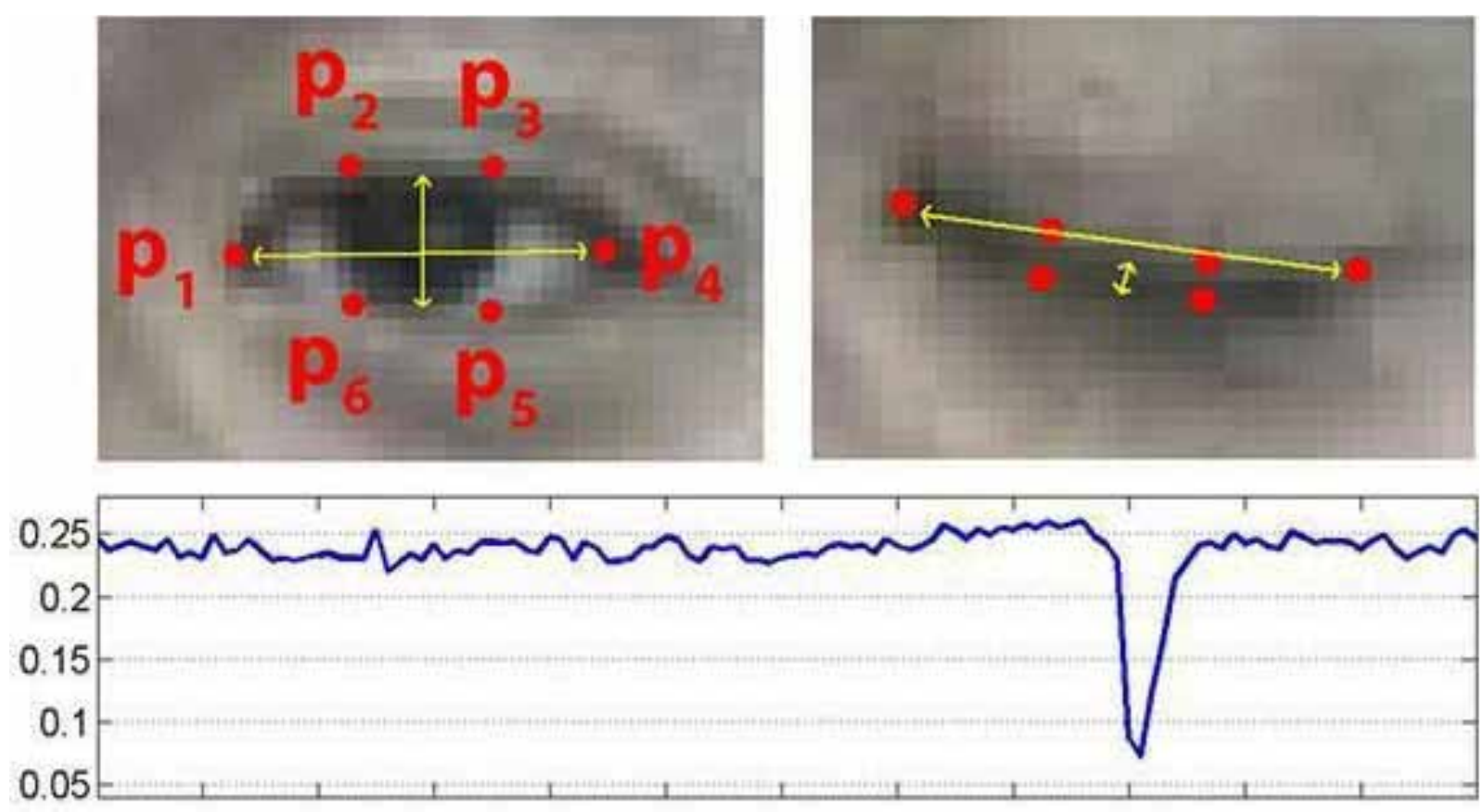

Fig. 3. Visualization of the eye aspect Ratio

Fig. 3 depicts the visualization of the eye aspect ratio when the eyes are open and close. As show in the figure the eye aspect ratio is constant when the eyes are open and is close to zero when the eyes are closed.

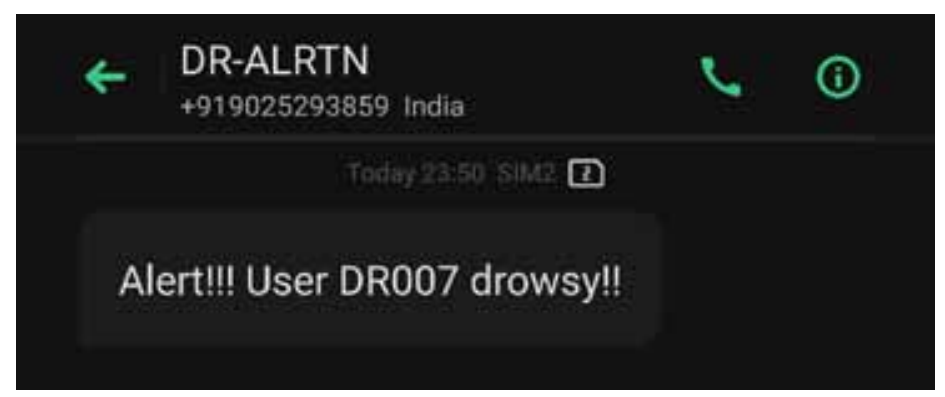

Fig. 4. SMS alert sent when user drowsy.

Fig. 4 shows the SMS sent by the system to the concerned person alerting that the user is drowsy. 


\section{Results and Observation}

The existing Drowsiness detection systems are made and focused on only driver drowsiness and doesn't provide any additional features to be used in other applications. Drowsiness detection can be used in other applications such as employee monitoring. The proposed system can be implemented in both driver drowsiness system and in employee monitoring with an additional feature which doesn't just alert the victim (the person being drowsy) but also the concerned person by sending an SMS.

\section{Conclusion}

Drowsiness is a very serious issue in many situations. The present systems mainly concentrate on driver drowsiness and only alerts the driver or the person drowsy. Our proposed system is a general-purpose drowsiness detection system which can not only be used for driver drowsiness detection but can also be used to check employee's activity and it can also be used to send SMS to the concerned person and alert the person who is drowsy. Our system is fairly straightforward. A camera is setup to find faces. After finding face we send the bounding box of face to find the eye region using facial landmark algorithm. The eye aspect ratio is computed and if the video segments average ear value exceeds the threshold value, we infer as the person is drowsy and send SMS to the concerned person and alert the person who is drowsy. This system can be further expanded with features like mobile use identification to find if the user is using phone and it can be improved to identify drowsiness in different cases such as, When the person is wearing shades.

Ethical Clearance: No ethical clearance required

\section{Source of Funding: Self}

Conflict of Interest: Nil

\section{References}

1. Tereza Soukupova', Jan C`ech. Real-Time Eye Blink Detection using Facial Landmarks. 21st Computer Vision Winter Workshop Luka Cehovin, Rok Mandeljc, Vitomir ` Struc (eds.) Rimske Toplice, Slovenia, February 3-5, 2016

2. B. Alshaqaqi, A. S. Baquhaizel, M. E. Amine Ouis, M. Boumehed, A. Ouamri and M. Keche. Driver drowsiness detection system. 2013 8th International Workshop on Systems, Signal Processing and their Applications (WoSSPA), Algiers, 2013, pp. 151155.

3. B.N. Manu. Facial features monitoring for real time drowsiness detection. 2016 12th International Conference on Innovations in Information Technology (IIT), Al-Ain, 2016, pp. 1-4.

4. W. Tipprasert, T. Charoenpong, C. Chianrabutra and C. Sukjamsri. A Method of Driver's Eyes Closure and Yawning Detection for Drowsiness Analysis by Infrared Camera. 2019 First International Symposium on Instrumentation, Control, Artificial Intelligence, and Robotics (ICA-SYMP), Bangkok, Thailand, 2019, pp. 61-64.

5. Tianyi Hong and Huabiao Qin. Drivers drowsiness detection in embedded system. 2007 IEEE International Conference on Vehicular Electronics and Safety, Beijing, 2007, pp. 1-5.

6. T. Danisman, I. Bilasco, C. Djeraba, and N. Ihaddadene. Drowsy driver detection system using eye blink patterns. In Machine and Web Intelligence (ICMWI), 2010, pp. 230-233.

7. Maria Anu V., R. Aroul Canessane, "Livestock Monitoring using RFID with R + Tree Indexing" in Biomedical Research in October 2016 Special Issue Pg: 1-4 ISSN:0970-938X

8. Jeyanthi P. and Jawahar Senthil Kumar V. (2010), "Image Classification by K-means Clustering", Published in the International journal of computational science and Technology, Research India Publications, Vol. 3, No. 1, pp.1-8, ISSN 0973-6107. 